\title{
Mutation in OsLMS, a gene encoding a protein with two double-stranded RNA binding motifs, causes lesion mimic phenotype and early senescence in rice (Oryza sativa L.)
}

\author{
Jerwin R. Undan ${ }^{1,2,3}$, Muluneh Tamiru ${ }^{1}$, Akira Abe ${ }^{2,4}$, Kentaro Yoshida $^{1}$, Shunichi Kosugi ${ }^{1}$, \\ Hiroki Takagi $^{1,2}$, Kakoto Yoshida ${ }^{1}$, Hiroyuki Kanzaki ${ }^{1}$, Hiromasa Saitoh ${ }^{1}$, Rym Fekih ${ }^{1}$, \\ Shailendra Sharma ${ }^{1}$, Jesusa Undan ${ }^{1}$, Masahiro Yano ${ }^{5}$ and Ryohei Terauchi ${ }^{1 *}$ \\ ${ }^{1}$ Iwate Biotechnology Research Center, Kitakami, Narita 22-174-4, Iwate 024-0003, Japan \\ ${ }^{2}$ United Graduate School of Agricultural Sciences, Iwate University, Morioka, Iwate 020-8550, Japan \\ ${ }^{3}$ Central Luzon State University, Science City of Muñoz, Nueva Ecija, 3120, Philippines \\ ${ }^{4}$ Iwate Agricultural Research Center, Narita 20-1, Kitakami, Iwate 024-0003, Japan \\ ${ }^{5}$ National Institute of Agrobiological Resources, Tsukuba, Ibaraki 305-8602, Japan
}

(Received 17 April 2012, accepted 14 June 2012)

The rice (Oryza sativa L.) lesion mimic and senescence (lms) EMS-mutant, identified in a japonica cultivar Hitomebore, is characterized by a spontaneous lesion mimic phenotype during its vegetative growth, an accelerated senescence after flowering, and enhanced resistance to rice blast (Magnaporthe oryzae). To isolate the OsLMS gene, we crossed the lms mutant to Kasalath (indica), and used mutant $\mathrm{F}_{2}$ plants to initially map the candidate region to about $322-\mathrm{kb}$ on the long arm of chromosome 2. Illumina whole-genome re-sequencing of the mutant and aligning the reads to Hitomebore reference sequence within the candidate region delineated by linkage analysis identified a $G$ to $A$ nucleotide substitution. The mutation corresponded to the exon-intron splicing junction of a novel gene that encodes a carboxyl-terminal domain (CTD) phosphatase domain and two double stranded RNA binding motifs (dsRBM) containing protein. By PCR amplification, we confirmed that the mutation causes splicing error that is predicted to introduce a premature stop codon. RNA interference (RNAi) transgenic lines with suppressed expression of $L M S$ gene exhibited the lesion mimic phenotype, confirming that the mutation identified in $L M S$ is responsible for the mutant phenotype. OsLMS shares a moderate amino-acid similarity to the Arabidopsis FIERY2/CPL1 gene, which is known to control many plant processes such as stress response and development. Consistence with this similarity, the lms mutant shows sensitivity to cold stress at the early growth stage, suggesting that $L M S$ is a negative regulator of stress response in rice.

Key words: double stranded RNA, lesion mimic, mutant screening, splicing error, whole-genome re-sequencing

\section{INTRODUCTION}

Mutants that show spontaneous cell death or necrotic lesions, which resemble the hypersensitive response (HR) caused by pathogen infection, in the absence of pathogens and abiotic stresses are called lesion mimic mutants (LMMs) (Moeder and Yoshioka, 2008). Although a substantial number of LMMs were identified in multiple plant species, including around 40 mutants in rice (Oryza sativa L.) (www.shigen.nig.ac.jp/rice/oryzabase/top/top.jsp), only

Edited by Toru Terachi

* Corresponding author. E-mail: terauchi@ibrc.or.jp few of the genes associated with the mutant phenotypes have been characterized so far (Moeder and Yoshioka, 2008; Qiao et al., 2010). LMMs show variation with respect to lesion size and color, as well as timing and conditions of lesion formation (Johal et al., 1995). Accordingly, LMMs are referred to either as initiation mutants, which show localized lesions of variable sizes that are constitutively formed, or as propagation mutants because they lack control over the rate and extent of cell death once it starts (for a review Lorrain et al., 2003). In rice, the lesions are usually reddish brown and dotted spot-like that mostly appear on the leaf surface, but are also occasionally formed on other plant parts. 
The first lesion mimic mutant identified in rice is the spotted leaf 1 (spl1), also called the Sekiguchi lesion ( $s l$ ), that displays spontaneous disease-like lesions in the absence of any pathogen, and confers resistance to multiple isolates of rice blast (Sekiguchi and Furuta, 1965). Other LMMs of rice such as spl11 (Yin et al., 2000) and spl28 (Qiao et al., 2010) also show either nonrace-specific or race-specific resistance to rice blast and bacterial blight. Although there has been no report showing a clear association between proteins encoded by LMM genes and the execution of programmed cell death (PCD) (Moeder and Yoshioka, 2008), LMMs have provided important tools for studying cell death resembling HR and unraveling PCD pathways in plants (Dangl et al., 1996). To this effect, many LMMs have been employed in the study of various aspect of PCD such as ROS formation (Jabs et al., 1996), the $\mathrm{Ca}^{2+}$ ion influx (Jurkowski et al., 2004), sphingolipid metabolism (Brodersen et al., 2002) and chlorophyll biosynthesis and catabolism (Ishikawa et al., 2001). However, most lesion mimic phenotypes are caused by physiological alterations regulated by environmental factors such as light (Arase et al., 2000) temperature (Noutoshi et al., 2005) and day-length (Ishikawa et al., 2001), and are not directly associated with defense responses (Liu et al., 2003).

Previous studies have shown that LMM genes encode proteins that belong to various functional groups, and examples include a membrane associated protein, an ion channel protein, a zinc-finger protein, a heat stress transcription, a U-box/Armadillo repeat protein as well as components involved in the biosynthesis/metabolic pathways of fatty acids/lipids, porphyrin and phenolic compounds (Qiao et al., 2010). Based on the identities of these genes, it has been suggested that the LMMs phenotype is the result of not only alternations in HR- response to pathogens but also due changes in various physiological pathways that are essential in plants response to both biotic and abiotic stimuli (Ishikawa et al., 2001; Qiao et al., 2010). Plants are exposed to both abiotic and biotic stresses that cause various physiological damage or disturbances in plants overall growth. The plant mechanisms coping these stresses include metabolic and structural adjustments, which involve genes that reside in different organelles and control the induction or suppression of specific proteins regulating stress responses (Suzuki and Mitler, 2006).

Senescence in plants, including leaf senescence, is a type of programmed cell death that is affected not only by endogenous development factors such as aging and hormones, but also by various environmental variables such as stress and nutrient supply (Nam, 1997). Accordingly, many stress related genes that are induced during senescence provide important clues regarding the molecular mechanism of initiation and development of leaf senescence. In this regard, detailed characterization of early senescence mutants is invaluable. In the present work, we identified and characterized a lesion mimic and early senescence (lms) mutant identified in a japonica cultivar Hitomebore and isolated the LMS gene, which encodes a protein that contains a carboxyl-terminal domaim (CTD) phosphatase domain and two doublestranded RNA binding motifs (RBM). LMS shares moderate amino-acid identity to the Arabidopsis FIERY2/ CPL1 gene, which is known to control many plant processes such as stress response and development (Koiwa et al., 2002; Xiong et al., 2002). To our knowledge, this is the first map-based cloning of a gene implicated in the formation of lesions and development of early senescence from the same protein family in rice. $l m s$ is sensitive to cold stress at the early growth stage, but shows enhanced resistance to rice blast, suggesting the possible involvement of $L M S$ is the regulation of stress responses in rice.

\section{MATERIALS AND METHODS}

Plant materials The lesion mimic and senescence (lms) mutant used in this study was identified in a mutant population of an elite japonica rice cultivar Hitomebore generated by ethyl-methanesulfonate (EMS) treatment. The mutagenesis protocol was provided elsewhere (Rakshit et al., 2010).

Generation of mapping population, linkage analysis and sequencing For linkage analysis, the mutant was crossed to the indica rice cultivar Kasalath and the resulting $\mathrm{F}_{1}$ plants were self-fertilized to generate $\mathrm{F}_{2}$ seeds. The segregation in $\mathrm{F}_{2}$ progeny was scored in the paddy field in the summer. A total of 60 polymorphic simple sequence repeat (SSR) markers, 5 markers randomly distributed on each rice chromosome, and $\mathrm{F}_{2}$ plants showing the mutant phenotype were used for rough mapping. Indel markers, containing 7-10 bp deletion either in Hitomebore or Kasalath genome, developed in this study were employed to further narrow-down the candidate region. To identify the causative mutation, the lms mutant was subjected to whole genome sequencing using an Illumina GAIIx sequencer (Illumina, San Diego, USA) and the reads within the candidate region delineated by linkage analysis were aligned to a reference sequence of Hitomebore, which was developed from the same population used for the mutagenesis, using the MAQ software (Li et al., 2008).

Construction of pANDA-LMS binary vector for RNAi analysis Total RNA was extracted from Hitomebore wild-type (WT) leaves using the Qiagen RNeasy mini kit according to the manufacturer's instructions (www.qiagen.com; Qiagen, Hilden, Germany) and treated with DNase to remove contaminating DNA. Two $\mu \mathrm{g}$ of total RNA were converted to complementary DNA (cDNA) 
with TOYOBO RTase (TOYOBO, Osaka, Japan). A 328bp sequence spanning the last exon and 3' UTR (untranslated region) of the $L M S$ gene was amplified with forward (5'-CACCAAGCCAGATTTTCCACGGGCA-3') and reverse (5'-TGGGTATCCACAAGGATGGGCA-3') primers using KOD-Plus DNA polymerase (www.toyobo.co.jp). The amplified fragment was sub-cloned into a Gateway pENTR/D-TOPO cloning vector (Invitrogen, Carlsbad, USA), transformed into competent Escherichia coli (DH5) cells, and was finally cloned into pANDA vector using LR recombination reaction. Nucleotide sequence of the construct was confirmed using the BigDye Terminator v3.1 Cycle sequencing Kit (Applied Biosciences, Foster City, USA). The binary vector, pANDA-LMS, was introduced into Hitomebore WT by Agrobacterium-mediated transformation.

Histochemical staining Fresh leaves were stained with 3,3'-diaminobenzidine (DAB) overnight. After staining, the chlorophyll was extracted by soaking the samples in $100 \%$ ethanol for $3 \mathrm{~h}$ or until the green pigment was totally removed. The samples were then examined using light microscope.

LMS gene expression analysis Total RNA was extracted from leaf tissue of RNAi transformants, including control plants, using the Qiagen RNeasy mini kit according to the manufacturer's instructions (www.qiagen.com) and contaminating DNA was removed by DNase treatment. Two $\mu \mathrm{g}$ of total RNA were converted to cDNA with RTase (TOYOBO). To quantify the mRNA expression levels, real-time PCR analysis was performed using SYBR green (QIAGEN) on StepOnePlus ${ }^{\mathrm{TM}}$ (Applied Biosystems) and the relative expression levels of each transcript were normalized using the OsActin1 gene. The primers used for RT-PCR were as follows: LMS-F (5'-GGGGGCTTCTTTAAGGACTTT-3'), LMS-R (5'-CGCAACATTCTCATCCTCTG-3'), Actin-F (5'CCCCCATGCTATCCTTCGT-3') and Actin-R (5'-GGCCGTTGTGGTGAATGAGT-3').

Phylogenetic analysis LMS and related amino acid sequences were identified using BLAST search for nonredundant proteins (http://blast.ncbi.nlm.nih.gov/). The deduced amino acid sequences were aligned using the ClustalW program version 2.0.3 provided in the Phylogeny.fr software (Dereeper et al., 2008) using the default parameters. Phylogeny.fr was also employed for phylogenetic analysis and tree rendering using the programs BioNJ (neighbor-joining) and TreeDyn 198.3, respectively. Bootstrap values were calculated based on 500 permutations.

Cold stress tolerance assay To determine the response of $l m s$ mutant to cold stress, WT and $l m s$ plants were ini- tially grown in soil under $16 \mathrm{~h}$ light and $8 \mathrm{~h}$ dark- photoperiods at $28 \pm 2^{\circ} \mathrm{C}$ for 30 days, and then incubated at $15^{\circ} \mathrm{C}$ for 5 days under the same photoperiod. After the cold treatment, plants were put back under the initial growing condition for an additional 5 days before data was collected.

Blast infection assay Four-weeks old $l m s$ and WT plants were inoculated with both compatible and incompatible strains of rice blast (Magnaporthe oryzae) using rice leaf blade spot inoculation test (for details refer to Kanzaki et al., 2002). For compatible (susceptible) interaction, the rice blast race Sasa2 (037) was used. Sasa2 transformed with AvrPii was used for the incompatible (resistance) interaction. The conidia concentration was adjusted to $5 \times 10^{5} \mathrm{ml}^{-1}$ and Tween 20 was added to a concentration of $0.5 \%$ before inoculation. Inoculated plants were incubated in $27^{\circ} \mathrm{C}$ growth chamber, and disease lesions were evaluated ten days after inoculation. Two independent experiments were carried to assess the response of $l m s$ to rice blast.

\section{RESULTS}

Phenotypic characterization of the $\mathbf{l m s}$ mutant The $l m s$ mutant was identified in $\mathrm{M}_{2}$ lines of the cultivar Hitomebore mutagenized by EMS treatment. Mutant plants grown under normal summer field conditions are characterized by reddish-brown necrotic lesions that are distributed over the entire leaf surface. The lesions were visible from about 30-40 days after sowing (DAS) but became severe around flowering time (Fig. 1A) mainly on the older lower leaves. Moreover, compared to the WT plants, the lms mutant exhibited rapid senescence after flowering (Fig. 1, B and C). This observation was similar to the finding reported for the rice spotted leaf mutant spl28 (Qiao et al., 2010). However, unlike the spl28 and blast lesion mimic (blm) (Jung et al., 2005) mutants that produced smaller seeds, the $l m s$ seeds were in general comparable to the WT seeds (Fig. 1D).

The reddish-brown lesions of the mutant were clearly visible from about 50 DAS, while no such lesions were observed on the WT plants (Fig. 2A). It has been known that $\mathrm{H}_{2} \mathrm{O}_{2}$ generated by various environmental and developmental stimuli act as a signaling molecule that regulate plant development, stress adaptation and PCD (Apel and Hirt, 2004). To check if the lesion development in lms involves $\mathrm{H}_{2} \mathrm{O}_{2}$ accumulation, we employed DAB staining that detects areas of brown precipitate upon its polymerization. In lms, we observed extensive DAB stain on leaf blade, indicating generation of excess of hydrogen peroxide in this mutant (Fig. 2B).

Map-based cloning of the OsLMS gene Of the $164 \mathrm{~F}_{2}$ plants obtained from the cross between $l m s$ and Kasalath, 
A

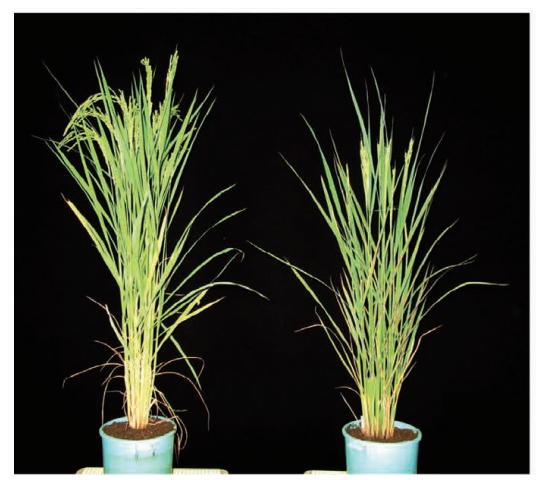

WT
B

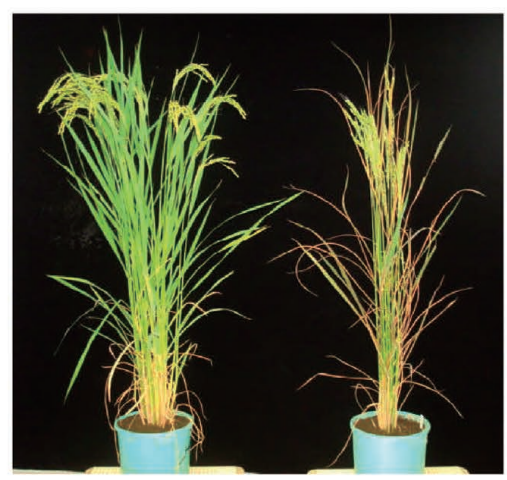

WT
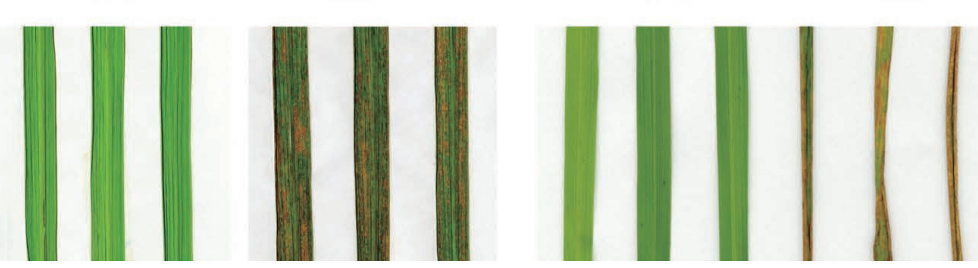

C

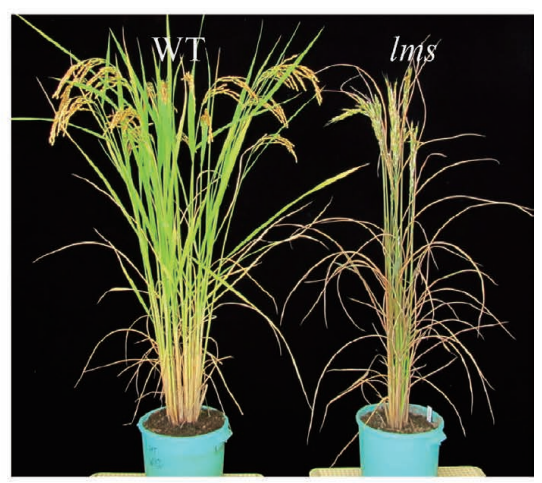

$\mathrm{D}$

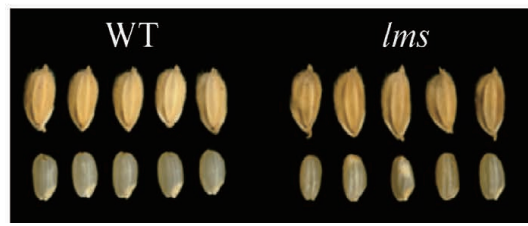

Fig. 1. The Oslms mutant shows lesion mimic phenotypes and rapid senescence following maturity. The gross morphology of WT and $l m s$ plants was compared at 95 (A), 105 (B) and 115 days after sowing (DAS) (C). (D) Seed and grain morphology of Hitomebore (WT) and $l m s$ mutant.
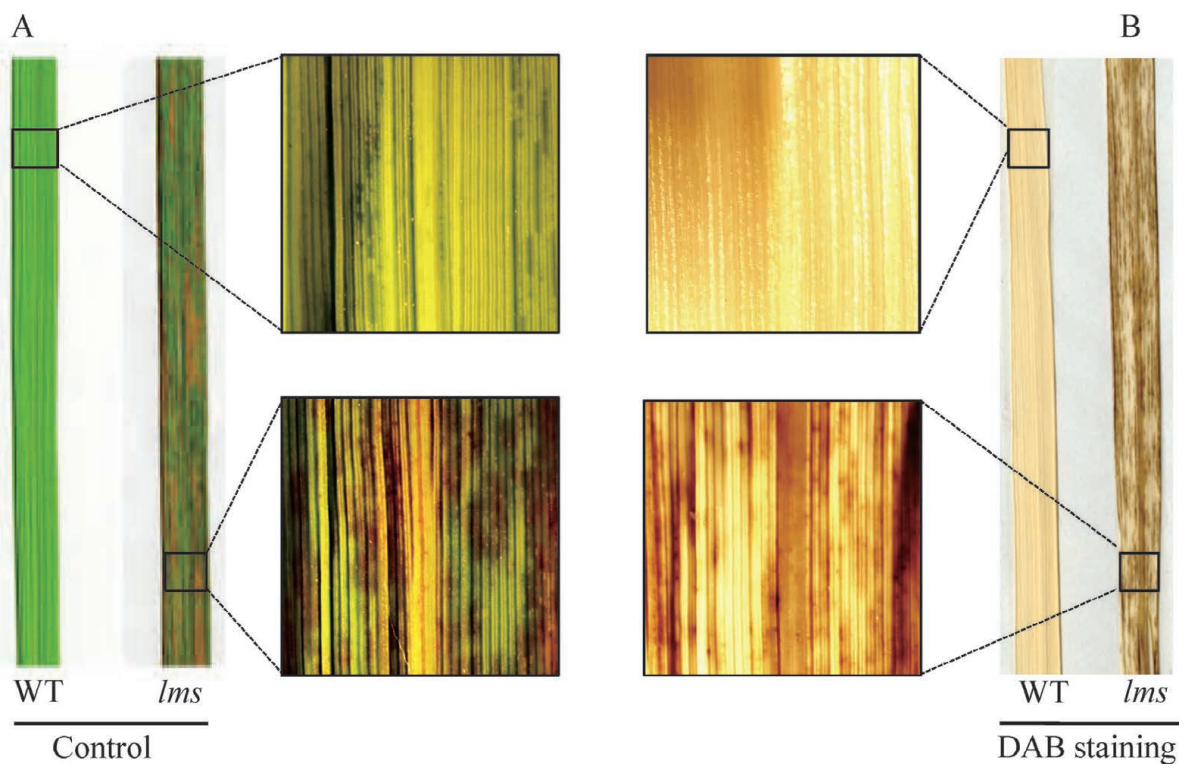

Fig. 2. DAB staining of leaves. (A) Leaves of WT and $l m s$ plants were collected at 50 DAS and (B) chlorophyll was removed by boiling the leaves in ethanol, and $\mathrm{H}_{2} \mathrm{O}_{2}$ was visualized as brown coloration by DAB (3,3' Diamino-benzedine) staining.

39 showed the lesion mimic phenotype in the paddy field. The segregation ratio conformed to 3:1 (Chi square test: $\chi^{2}=0.13, \mathrm{~ns}$ ) indicating that a single recessive gene controls the lms phenotype. The LMS locus was identified by a combination of linkage analysis (using SSR and Indel markers for the 39 mutant-type $\mathrm{F}_{2}$ plants) and whole-genome re-sequencing of the mutant. The locus was initially mapped to about $770-\mathrm{kb}$ region on the long arm of chromosome 2 between two SSR markers
RM13637 and RM13670 (Fig. 3A). We then developed indel markers within the candidate region using Hitomebore and Kasalath reference sequences to further narrow down the locus to about 332-kb between the indel markers Indel-26351146 and Indel-26683091. Within this region, Indel-26443890 was closely linked to the $L M S$ locus (Fig. 3A). Sequences of the Indel markers used for the linkage analysis are provided in Table 1.

The $332-\mathrm{kb}$ candidate region identified by linkage anal- 
A

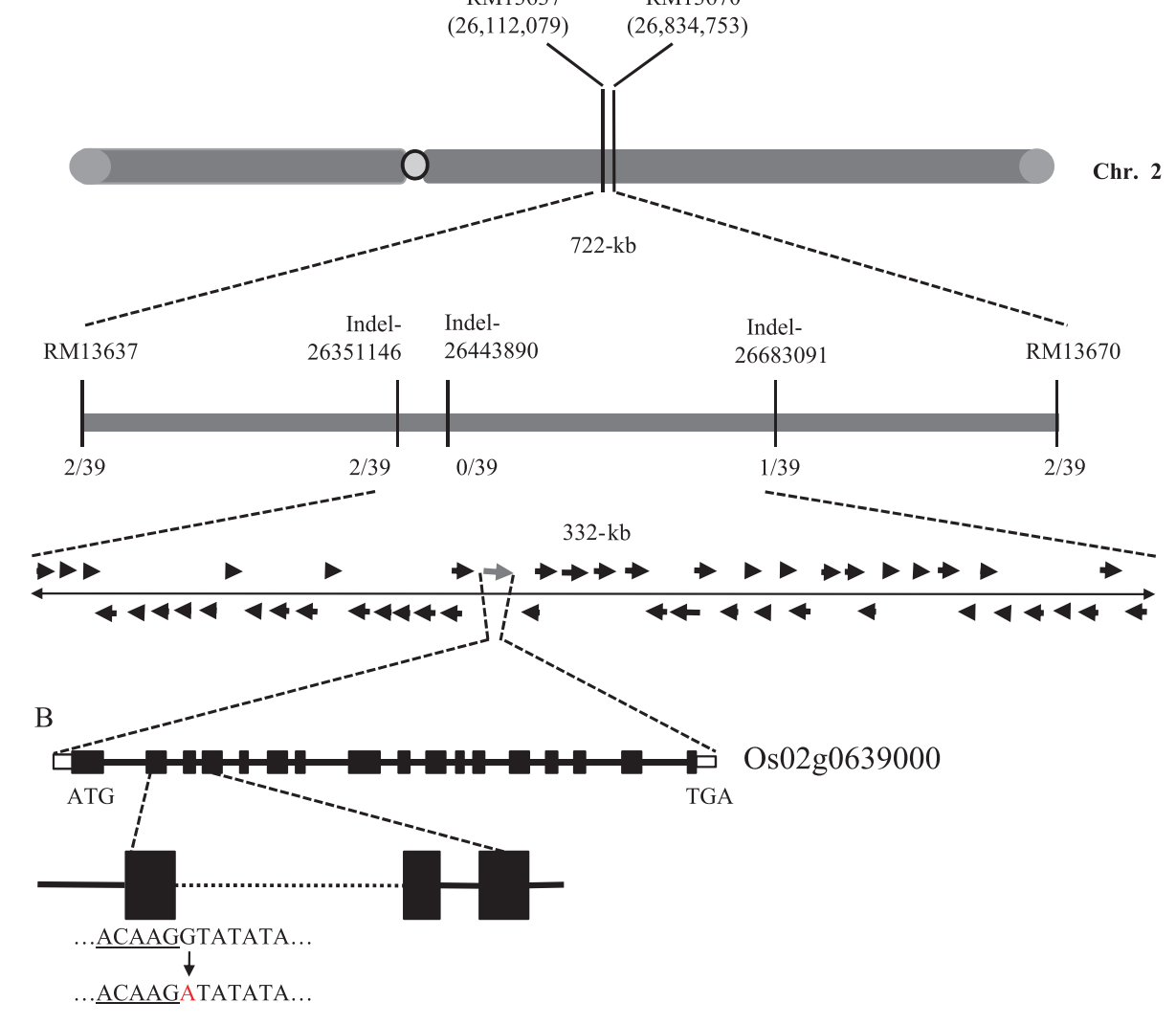

$\mathrm{C}$

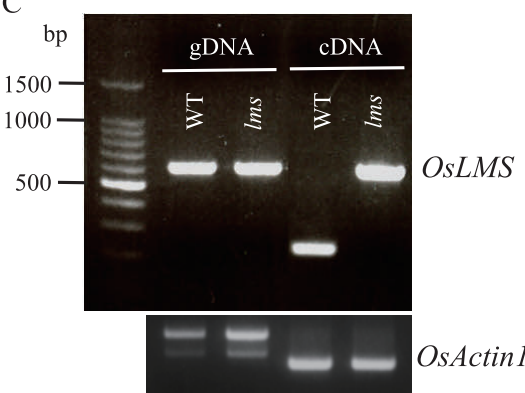

Fig. 3. Map-based cloning of the OsLMS gene. (A) The candidate locus was initially mapped to about 722 -kb region between simple sequence repeat (SSR) markers RM13637 and RM13670 on the long arm of chromosome 2, which was further narrowed to about 322-kb between indel markers Indel-26351146 and Indel-266839091 using additional mapping population. Arrows represent the 47 predicted genes located within the 322 -kb candidate region, and gene orientation is given by the direction of each arrow. (B) Whole genome sequencing of $l m s$ and comparison of the sequence to Hitomebore reference sequence revealed a single base substitution (G to $A)$ in the intron-splicing site of Os02g0639000 (OsLMS). The structure of $L M S$ gene with 17 exons and 16 introns is represented with black boxes and lines, respectively. Open boxes indicate untraslated regions (UTRs). The location of the mutation at the splicing junction of the second exon and the second intron is indicated. The underlined sequences belong to exon 2. (C) Splicing error in the lms gene transcript. PCR amplification of genomic DNA (gDNA) and complementary DNA (cDNA) was performed for WT and lms mutant plants using primes designed in the $2^{\text {nd }}$ and $3^{\text {rd }}$ exons and spanning the site of the point mutation, showing that $2^{\text {nd }}$ intron is not spliced in lms. PCR amplification of OsActin1 gene served as a control for removal of gDNA from cDNA preparation.

Table 1. Sequences of Indel markers used for linkage analysis

\begin{tabular}{ccc}
\hline \hline Primer name & Forward & Reverse \\
\hline Indel-26351146 & TACGTGGTGTCCTCGCTACA & TCCCTATAACGCGCTCACAT \\
Iddel-26683091 & GTACCGAATTGGGGTGTGAC & GCCATCCGATTATTGTGTCG \\
Indel-26443890 & CATGAAAGGCACAAGAACGA & AGGTGCCACACTGTCTCAGTT \\
\hline
\end{tabular}


ysis is predicted to contain about 47 open reading frames (ORFs). To identify the causative mutation, we sequenced the $l m s$ mutant using a single lane of Illumina GAIIx sequencer to a depth of $16 \times$ that gave about $94.5 \%$ coverage of the rice genome. The reads within the candidate region delineated by linkage analysis were aligned to a reference sequence of Hitomebore using the MAQ software. We earlier developed the Hitomebore draft reference sequence from the same population used for the mutagenesis (Abe et al., 2012). The sequence comparison identified only a single homozygous SNP resulting in nucleotide substitution from $\mathrm{G}$ to $\mathrm{A}$ that corresponded to the splicing junction at the end of second intron of the Os02g0639000 locus (Fig. 3B).

Splicing junction mutations are know to cause splicing error during transcription, which may cause either mRNA or protein degradation leading to loss of function

A

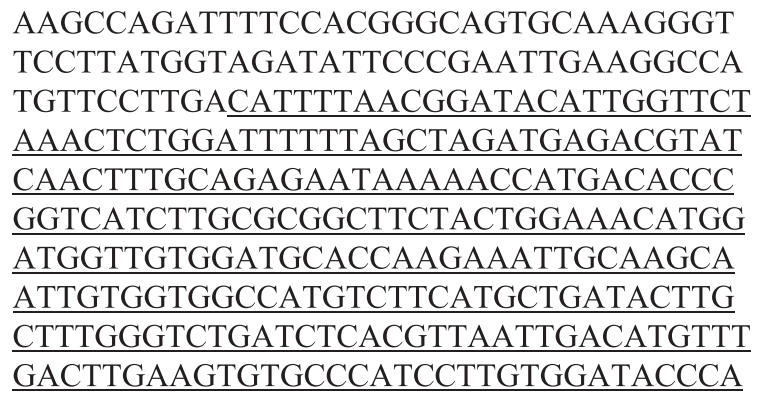

$\mathrm{C}$

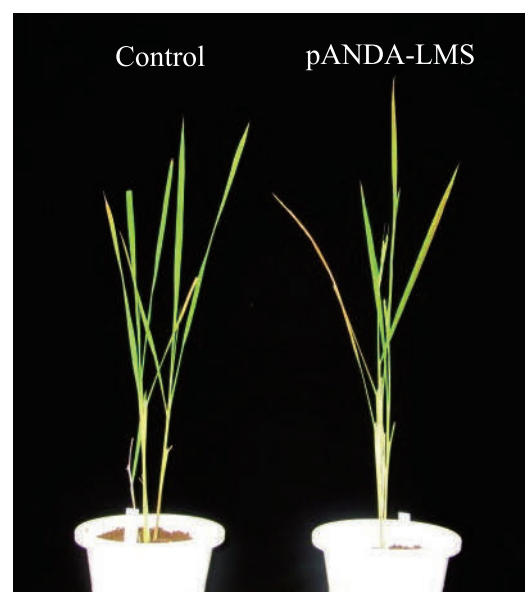

$\mathrm{D}$

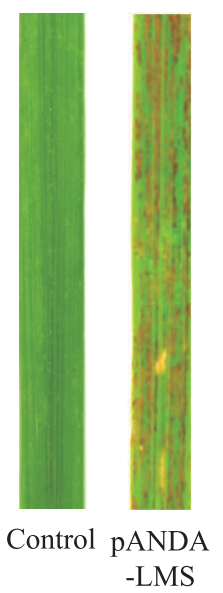

of genes. To verify whether the mutation detected in Os02t0639000 leads to splicing error, we prepared genomic DNA (gDNA) and cDNA both from WT and mutant plants and performed PCR amplification using gene specific primers designed within the $2^{\text {nd }}$ and $3^{\text {rd }}$ exons covering the site of mutation and including the $378 \mathrm{bp} 2^{\text {nd }}$ intron. In both the mutant and WT, the expected $614 \mathrm{bp}$ fragment was amplified from the gDNA. While PCR amplification from WT cDNA gave the expected $236 \mathrm{bp}$ product, it produced the full length $614 \mathrm{bp}$ fragment that included the $378 \mathrm{bp}$ intronic sequence in the mutant, suggesting splicing error (Fig. 3C). The splicing error is predicted to introduce a premature stop codon with in the intronic sequence. Using OsActin gene as a control, we verified that the cDNA used for the amplification is free from gDNA contamination.

B
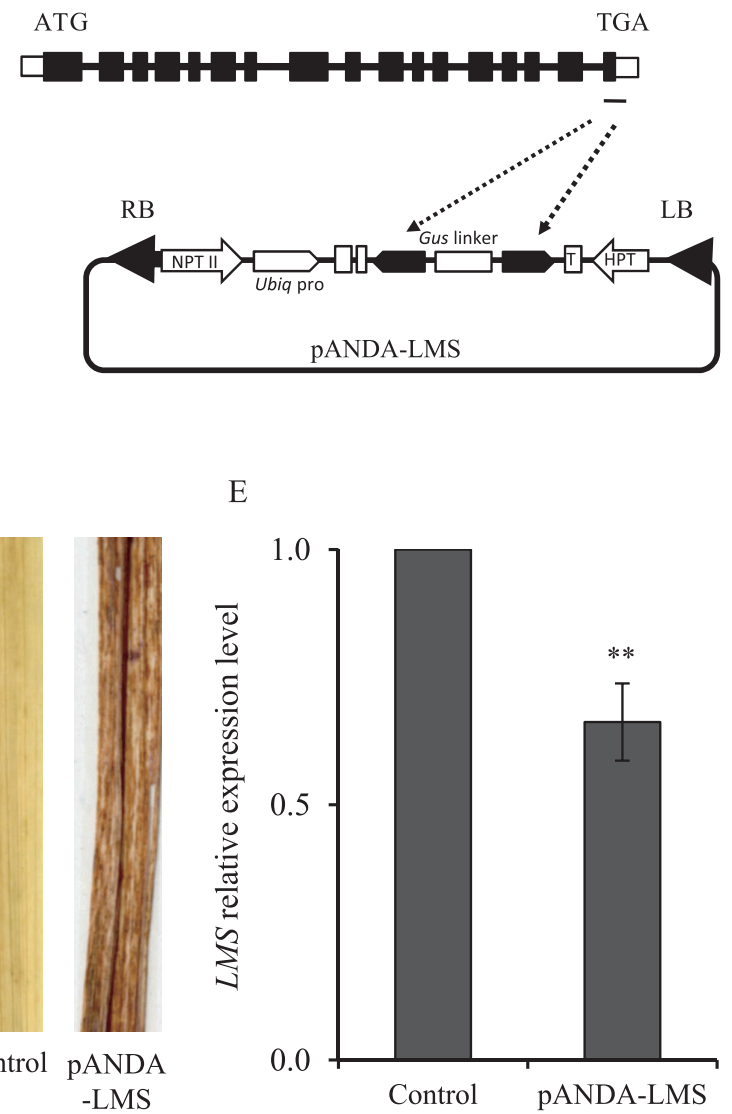

Fig. 4. RNAi mediated silencing of the OsLMS. (A) The $328 \mathrm{bp}$ sequence spanning the last exon and $3^{\prime}$ UTR region $O s L M S$ used for RNAi analysis. The region corresponding to the $3^{\prime}$ UTR region is underlined. (B) Schematic diagram of the RNA interference vector pANDA-LMS showing the $328 \mathrm{bp} l m s$ cDNA fragment inserted in an inverted orientation. (C) Comparsion of the control plant with empty vector and the RNAi plant harboring pANDA-LMS. RNAi plants showed lesion mimic phenotype similar to $l m s$ plants. (D) Leaves of the RNAi transformant before and after chlorophyll extraction and DAB staining. (E) Real time PCR analysis of the OsLMS gene expression levels. OsLMS transcripts were normalized to the levels of OsActin, and the expression level in the RNAi plant (pAND-LMS) was determined by setting the expression in the control plant to 1 . Values are the mean \pm SD of three replicates. Asterisks indicate significant differences (Student's $t$ test, $* * P<0.01$ ). The same RNAi line shown in Fig. 4C was used both for DAB staining and qRT-PCR analysis. 
RNAi analysis To check if the mutation detected in Os02t0639000 is responsible for the abnormal phenotypes of $l m s$, a 328 bp Os02t0639000-specific fragment was amplified from cDNA of Hitomebore (Fig. 4A) to construct an RNAi vector, pANDA- $L M S$ that was used to transform calluses obtained from Hitomebore WT (Fig. 4B). Twentyfour independent $\left(\mathrm{T}_{0}\right)$ transgenic plants were obtained. Of these, 14 showed lesion mimic phenotype similar to the lms mutant (Fig. 4C). However, there was variation among the transgenic lines with respect to the extent of the lesion mimic phenotype (data not shown). DAB staining revealed browning of leaves of RNAi plants, indicating hydrogen peroxide accumulation (Fig. 4D). Quan- titative RT-PCR analysis confirmed that the LMS transcript in $\mathrm{T}_{0}$ plants was significantly reduced $(\mathrm{P}<0.05)$ compared to the transformants harboring the empty vector (Fig. 4E). Taken together, our findings suggest that Os02t0639000 is the LMS gene.

OsLMS encodes a double stranded RNA binding domain (dsRBD) containing protein The LMS gene is composed of 17 exons and 16 introns (Fig. 4B), and contains a catalytic carboxyl terminal phosphatase domain (CPD) and two double stranded RNA binding motifs (Fig. 5). The gene encodes a protein that belongs to a double stranded RNA binding domain (dsRBD) containing pro-

\begin{tabular}{|c|c|c|}
\hline LMS & 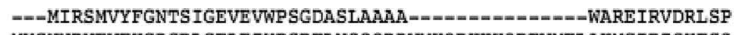 & \\
\hline FIERY2/AtCPL1 & MYSNNRVEVFHGDGRLGELE IYPSRELNQQQDDVMKQRKKKQREVMELAKMGIRISHFSQ & \\
\hline & 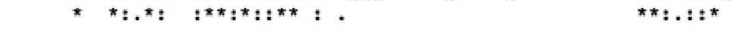 & \\
\hline LMS & PSERCQPLAVMHTVAVGARCLVMESRLPKAADEPPQPLVAMHAACLKENKTAVVPLGEEE & \\
\hline FIERY2/AtCPL1 & SGERCPPLAILTTISSCGLCFKLEASPS----PAQESLSLFYSSCLRDNKTAVMLLGGEE & \\
\hline & 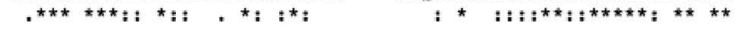 & \\
\hline LMS & LHLVAMTSRRNLTNHACFWGYKVPFGLYNSCLTMLNLRCLGIVFDLDETLIVANTTRSFE & \\
\hline FIERY2 / AtCPL1 & LHLVAMYSENIKNDRPCFWAFSVAPGIYDSCLVMLNLRCLGIVFDLDETLVVANTMRSFE & \\
\hline & 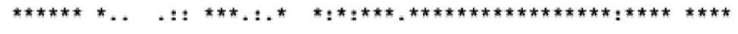 & \\
\hline LMS & DRIDALQRKLSNETDPQRINGMIAEIKRYQDDKSII KQYIEGDQVYDDGKMYKVQPELVF & \\
\hline FIERY2 /AtCPL1 & DKIDGFQRRINNEMDPQRLAVIVAEMKRYQDDKNLI KOYIESDOVVENGEVIKVOSEIVH & \\
\hline & 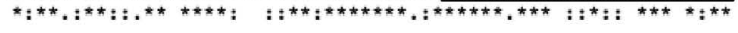 & \\
\hline LMS & PLSDNHQPMTRPVIRLQEKNIILTRINPLIRDTSVLVRLRPAWEDLRSYLIARGRKRFEV & \\
\hline FIERY2/AtCPL1 & ALSDNHOPLVRPLIRLOEKNIILTR INPMIRDT SVLVRMRPSWEELRSYLTAKGRKRFEV & C-terminal \\
\hline & 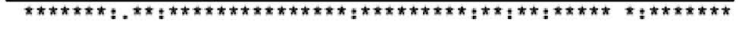 & Phosphatase \\
\hline LMS & YVCTMAERDYALEMWRLLDPDSRLINSVQLNDRMVCVKSGLRKSLLNVFHDGSCHPGMAI & Domain \\
\hline FIERY2/AtCPL1 & YVCTMAERDYALEMWRLIDPEGNLINTNDLLARIVCVKSGFKKSLFNVFLDGTCHPKMAI & \\
\hline & 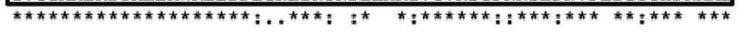 & \\
\hline LMS & VIDDRLKVWDEKDQSRVHVVPAFTPYYAPQ EANCSIPVLCVARNVACNVRGGFFKDFDE & \\
\hline FIERY2/AtCPL1 & VIDDRLKVWDEKDQPRVHVVPAFAPYYSPO EAA-ATPVLCVARNVACGVRGGFFRDFDD & \\
\hline & 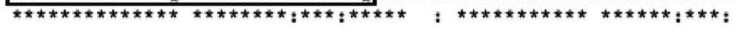 & \\
\hline LMS & GLLPRISNVFYEDEINEIPSAPDVGNYLISEDENVAAVNGNRDPLAFDGMADAEVERRMK & \\
\hline FIERY2/AtCPL1 & SLLPRIAEISYENDAEDIPSPPDVSHYLVSEDD-TSGLNGNKDPLSFDGMADTEVERRLK & \\
\hline & 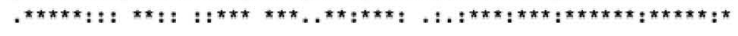 & \\
\hline LMS & EASGNAOAFTTTAAN--------FVMPVLPG---1-----ONFVSSSVAPVAPSLGMVPLSN & \\
\hline FIERY2/AtCPL1 & EAISASSAVLPAANIDPRIAAPVQF PMASASSVSVPVPVQVVQQAIQPSAMAFPSIPFQQ & \\
\hline & 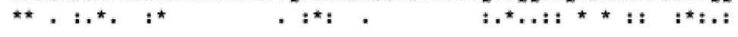 & \\
\hline LMS & NQGPPPFTQPVAQLSLSDPLQGSPAREEGEVPESELDPDTRRRLLILQHGQDTRDPTPP- & \\
\hline FIERY2/AtCPL1 & PQQPTSIA--KHLVPSEPSLQSSPAREEGEVPESELDPDTRRRLLILQHGQDTRDPAPSE & \\
\hline & 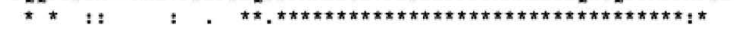 & \\
\hline LMS & --LPAVPPVQVPVPPVQPHGNWF PVEDGMNPNNLNRG-SAGFPLESETMHYDKKQLPHPF & \\
\hline FIERY2 /AtCPL1 & PSF PQRPPVQAPP SHVQSRNGWF PVEEEMDPAQIRRAVSKEYPLDSEMIHMEKHRPRHPS & \\
\hline & 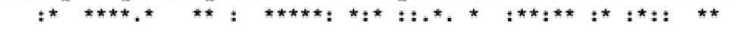 & \\
\hline LMS & FHG-GENPISSDRFSYQNQRYPSQLPHSEDHRVLNHAPSRYRSF PGEELATRHVSSSQR & \\
\hline FIERY2/AtCPL1 & FFSKIDNSTQSDRMLHENRRPPKESLRR-DEQLRSNNNLPDSHPFYGEDASWNQSSSRNS & \\
\hline & 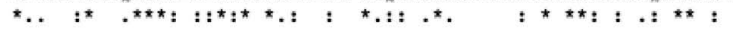 & \\
\hline LMS & NNQIVPGQHFARHAG SAGILEEIAMKCGSKVEYRSALCDTADLQFSIEVWIVGEKVGEG & Double stranded \\
\hline FIERY2/AtCPL1 & DLDFLPERS-VSATE SADVLHGIAIKCGAKVEYKPSLVSSTDLRFSVEAWLSNOKIGES & RNA \\
\hline & 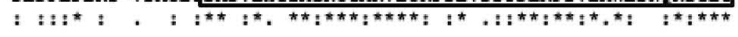 & Binding \\
\hline LMS & IGRTRKEAQCQAAEISLRNLA IKYLSSDPNKMTDMKEN------GFGSNTNIFGYPGN & Domain \\
\hline FIERY2 / AtCPL1 & IGKSRREALHKAAEASIONLA GYYMRANGDPGPSHRDATPFTNENISMGNANALNNOPFA & \\
\hline & 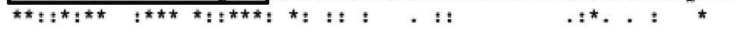 & \\
\hline LMS & SRDDVLPIASTSEETRFVKMGENNSRKAG O SIAALKELCTAEGYNLVFQARTSPDS--SV & Double stranded \\
\hline FIERY2/AtCPL1 & RDETALPVSSRPTDPR-----LEGSMRHTdSITALRELCASEGLEMAFQSQRQLPSDMVH & RNA \\
\hline & 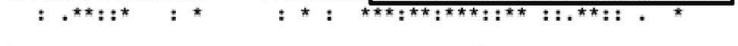 & Binding \\
\hline LMS & GKEAYAEVEVCGQILGNGVGITWEDAKLQAADEALGMLFSMLGPLAQKRSSSPRSLAPSF & Domain \\
\hline FIERY2/AtCPL1 & RDELHAOVEIDGRVVGEGVGSTWDEARMOAAERALSSVR SMLGQPLHKRQGSPRSFGGMS & \\
\hline & 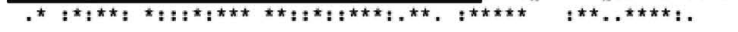 & \\
\hline LMS & DKRFKPDFPRAVQRVPY-GRYSRIEGHVP & \\
\hline FIERY2 /AtCPL1 & NKRLKPDFQRSLQRMPSSGRYS=------- & \\
\hline & $: * \pi: * * \pi * *: 1: * \pi: * \pi * \pi *$ & \\
\hline
\end{tabular}

Fig. 5. OsLMS encodes a double stranded RNA binding domain (dsRBD) containing protein that shares a moderate amino acid sequence similarity to the Arabidopsis FIERY2/AtCPL1 gene. The carboxyl-terminal phosphatase domain and the two double stranded RNA binding motifs are indicated. 
tein family. The LMS protein shares moderate amino acid similarity with the Arabidopsis FIERY2/CPL1 and its putative homolog in rice OsCPL1 (Xiong et al., 2002; Koiwa et al., 2002) (Fig. 5). The Arabidopsis CPL1 is known to negatively regulate the expression of stressresponsive genes and to modulate $\mathrm{ABA}$ response in Arabidopsis.

BLAST search indicated that LMS like proteins are plant specific. Phylogenetic analysis of LMS like proteins from higher plants based on the neighbor joining method revealed a clear separation between CPL1 and CPL2 like proteins (Fig. 6). The CPL1 like proteins from monocots further form a separate clade from their orthologs in dicots, while LMS and its homologs are clearly separated from the Arabidopsis CPL1 clade as well as rice OsCPL1 clade.

lms is sensitive to cold stress Based on cold tolerance assay, we confirmed that lms is sensitive to cold. When plants were moved back to normal growing conditions after the cold treatment, about $78 \%$ of WT plants showed full recovery from the stress, while only $21 \%$ of $l m s$ plants recovered (Fig. 7). This result suggests that the mutant is susceptible to low temperature injury, similar to an observation made in fiery 2 mutant during cold stress assay (Xiong et al., 2002). The lms mutant shows poor root growth compared to the WT plants (Fig. 7). It is possible that this poor root growth of the mutant contributed to its sensitivity to cold stress. Water absorption by roots is significantly reduced by exposure of seedlings to low temperature leading to water deficiency in the plant, and this effect would

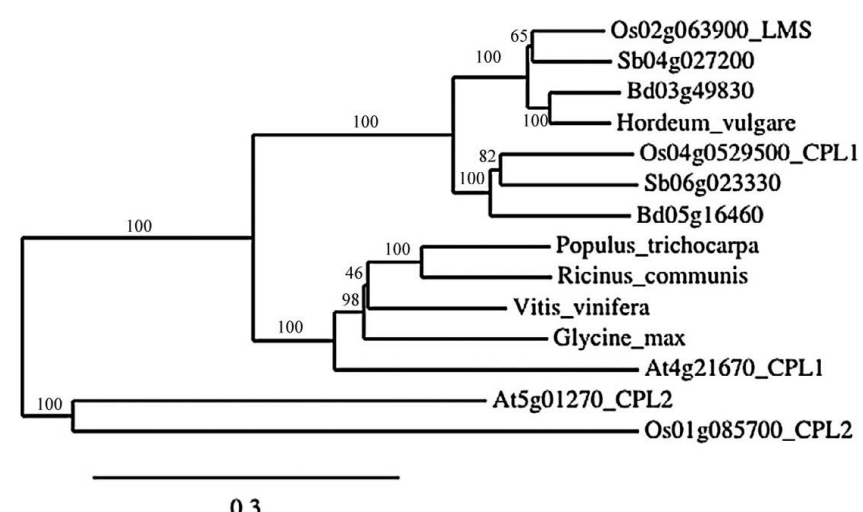

Fig. 6. Phylogenetic relationships of LMS homologs in plants as shown by a neighbour-joining (NJ) unrooted tree. GenBank accession numbers: Oryza sativa [Os02g063900_LMS (NP 001047536), Os04g0529500_CPL1 (CAD41201), Os01g085700 _CPL2 (BAB63701)], Sorghum bicolor [Sb06g023330 (XP_ 002446830), Sb04g027200 (XP_002452510)], Hordeum vulgare (BAJ93585), Brachypodium distachyon [Bd03g49830 (XP 003569958), Bd05g16460 (XP_003580187)], Glycine max (XP_ 003529311), Vitis vinifera (CAN72816), Populus trichocarpa (XP_002305017), Ricinus communis (XP_002519032), Arabidopsis thaliana [At4g21670_CPL1 (NP_193898), At5g01270_PL2 (NP_ 195747)].
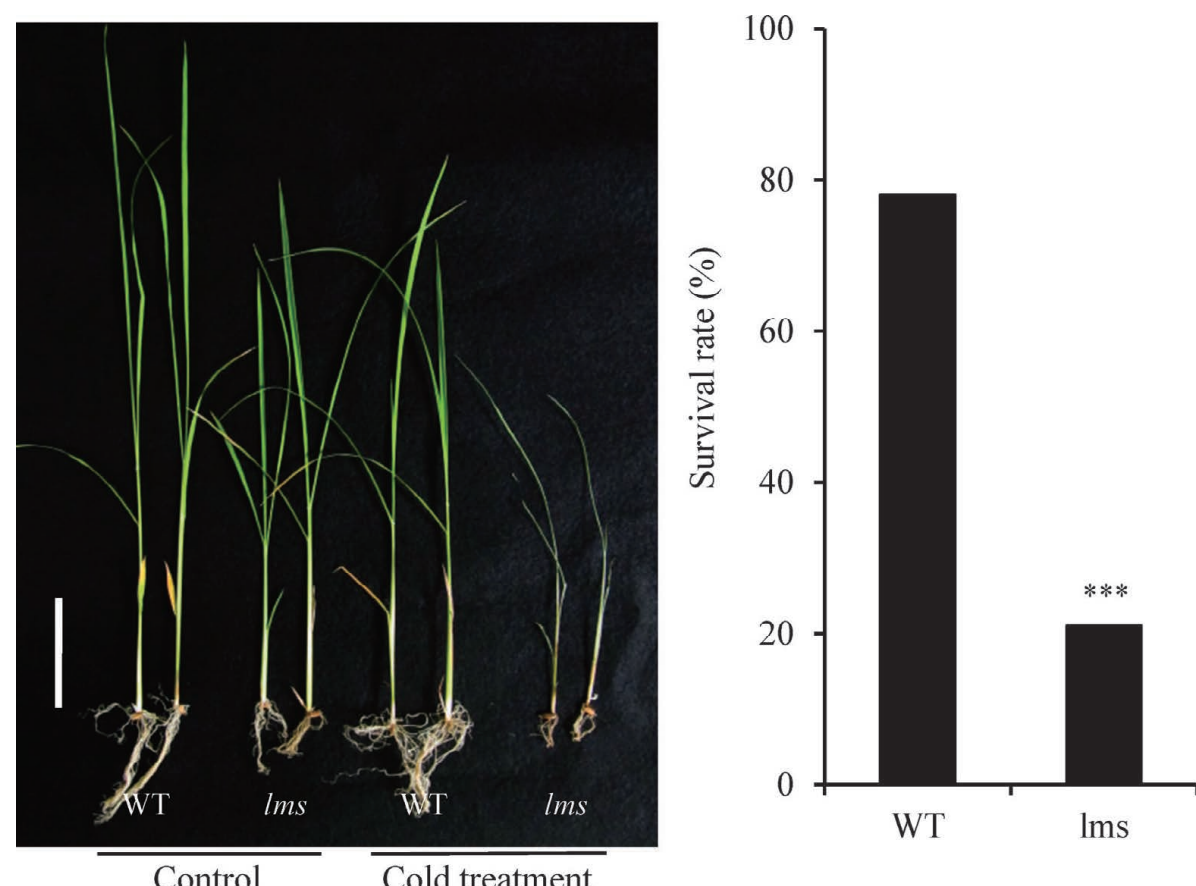

Fig. 7. Morphology of WT and $l m s$ plants before and after cold treatment. The plants were initially grown at $28^{\circ} \mathrm{C}$ for 30 days, then incubated at $15^{\circ} \mathrm{C}$ for 5 days, and finally moved back to $28^{\circ} \mathrm{C}$ for an additional 5 days before data collection. The control plants were kept at $28^{\circ} \mathrm{C}$. Bar represents $5 \mathrm{~cm}$. Asterisks indicate significant differences $(t$-test, $* * * P<0.001)$. 
A

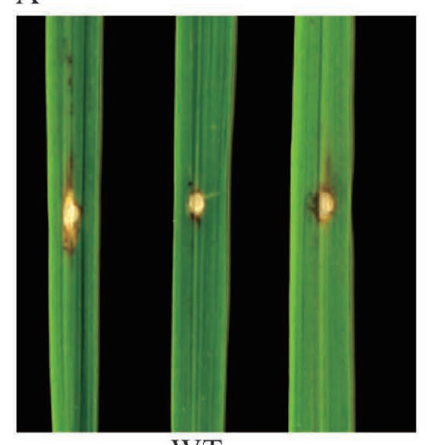

WT

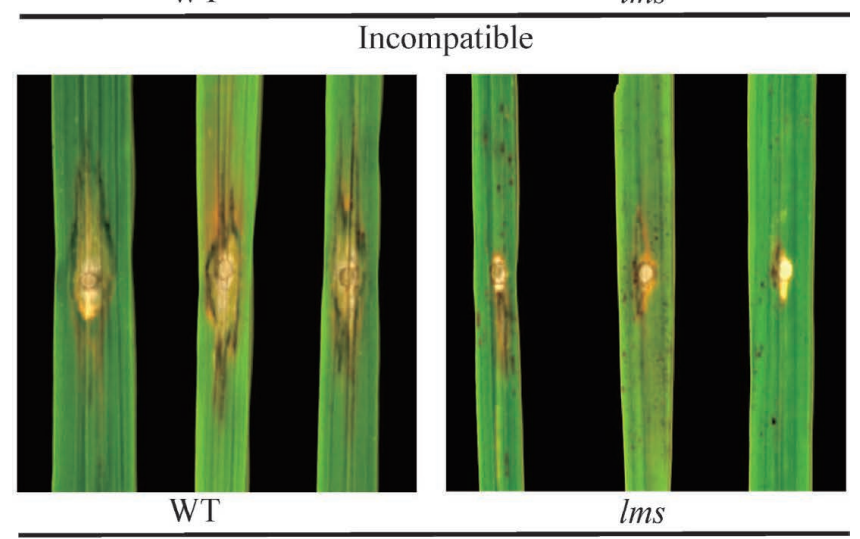

Compatible

B

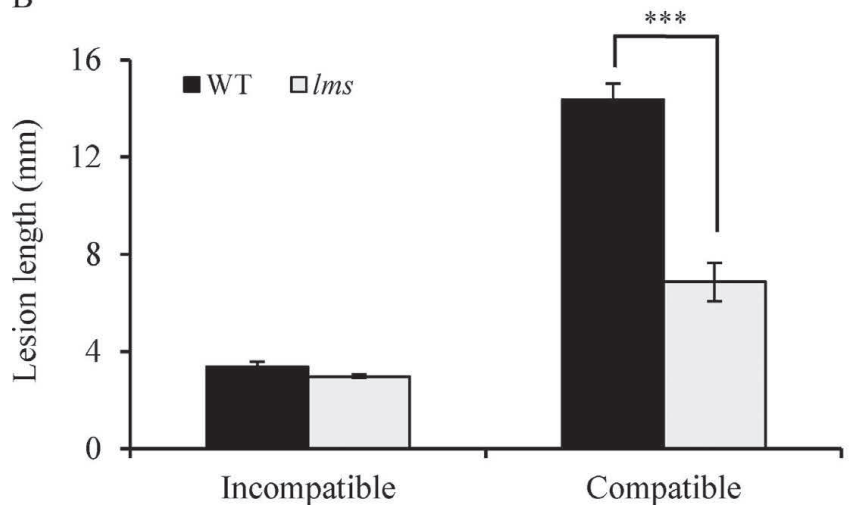

Fig. 8. The enhanced resistance of $l m s$ mutant to rice blast confirmed by a leaf blade inoculation assay using both incompatible (Sasa2, race 037.1-Pex33) and compatible (Sasa2, race 037) races of $M$. oryzae. (A) Phenotypes of the lesions observed. (B) A histogram showing the lesion size for WT Hitomebore and $l m s$ plants for both the incompatible and compatible interactions. Error bars represent standard error values $(n=29)$. Asterisks indicate significant differences (Student's $t$-test, ${ }^{* * *} P<0.001$ ).

be more enhanced if root development were already poor.

The lms plants exhibit enhanced resistance to rice blast Some LMMs show enhanced resistance to fungal and bacterial pathogens, suggesting they may represent steps associated with pathogen infection-triggered response pathway (Takahashi et al., 1999; Yin et al.,
2000; Wu et al., 2008). To check if the $l m s$ lesion mimic phenotype is also related to resistance response, we performed inoculation test using both compatible and incompatible strains of $M$. oryzae. As expected, both the WT and $l m s$ plants were resistant to the incompatible race of rice blast (Fig. 8). Upon inoculation with the compatible race however, WT plants were clearly susceptible, while the lms plants showed enhanced resistance. Similar results were obtained in two independent experiments.

\section{DISCUSSION}

In the present study, we report the identification of a rice $l m s$ mutant that is characterized by reddish-brown lesions, which first appear at about 30 days from sowing, and accelerated senescence after flowering. We employed a combination of linkage analysis and whole genome sequencing to isolate the $L M S$ gene. Using $\mathrm{DAB}$ staining, we detected areas of brown lesions in the mutant leaves that revealed $\mathrm{H}_{2} \mathrm{O}_{2}$ accumulation, suggesting that there is an oxidative burst leading to cell death in the mutant. The mutant is sensitive to cold, which means the $L M S$ gene is likely involved in stress signaling mechanism or other regulatory pathways involving stress responses such as cold tolerance in rice.

The LMS gene encodes a protein with a catalytic CTD phosphatase domain and two double stranded RNA binding motifs (dsRBM). The CTD phosphatase domain is involved in the dephosphorylation of the CTD of the largest subunit of RNA polymerase II (RNAP II), which consists of an evolutionary conserved heptapeptide consensus sequence Tyr-Ser-Pro-Thr-Ser-Pro-Ser as the pivotal phosphorylation target, thereby regulating transcription of target genes (Dahmus, 1996; Ji et al., 2010). RNAP II is a core component of the transcription complex and catalyzes mRNA synthesis, and is thus involved in the regulation of various mRNA maturation processes or gene transcription such as capping, splicing, and polyadenylation (Hirose and Manley, 2000; Xiong et al., 2002). The recently reported CTD phosphatase like gene in rice is involved in cell differentiation, and controls abscission layer development and seed shattering (Ji et al., 2010). Of the more than 20 genes in Arabidopsis genome that encode CTD-phosphatase like domain, only four (AtCPL1-AtCPL4) have been isolated and characterized in detail (Bang et al., 2006; Koiwa et al., 2002, 2004; Ueda et al., 2008; Xiong et al., 2002). In addition to the CTD phosphatase domain, AtCPL1 and AtCPL2 harbor two and a single double-stranded RNA binding motifs (DRM) in their C-terminus, respectively (Ueda et al., 2008). AtCPL1 is known to control expression of the DRE/CRT (drought-responsive/C-repeat) class of genes that regulate stress (cold and salt) and auxin response (Koiwa et al., 2002; Xiong et al., 2002). Likewise, AtCPL2 is involved in the regulation of plant growth, 
stress and auxin response (Ueda et al., 2008).

So far, there is no report on the involvement of genes encoding dsRBM family protein in the formation of lesion mimic or development of early senescence in plants. Several dsRBM proteins have biochemical roles in transcription, RNA processing, mRNA localization and translation, and function in a diverse range of critically important roles in the cell such as defense systems (Tian et al., 2004). BLAST analysis shows $L M S$ shares a moderate amino acid similarity with the Arabidopsis CPL1/FIERY2 gene, which regulates several abiotic stress responsive genes and show altered physiological responses to stress and ABA treatment in the fiery2 mutant (Xiong et al., 2002). Our result demonstrated that the lms mutant is susceptible to cold stress. This indicates to the possible involvement of $L M S$ in stress response such as cold. However, additional studies are needed to verify this response. The enhanced resistance response of $l m s$ to blast fungus confirmed that the spontaneous lesion mimic phenotype of the mutant is related to defense responses. This finding confirms previous reports from multiple crops species that established the link between the formation of lesion mimic phenotypes in several mutants and the activation of defense responses to diverse pathogens (Takahashi et al., 1999; Yin et al., 2000; Mizobuchi et al., 2002; Wu et al., 2008).

Although the identification of the lms mutant and the isolation of the corresponding $L M S$ gene represent an important addition to the current knowledge on LMMs, physiological and molecular analyses are further required to characterize the mutant in detail and decipher the gene function. Additional studies on the response of the mutant to environmental stimuli such as light and humidity, as well as response to different phytohormones are also being considered for future investigation on this mutant.

The first author was supported by the Japanese Government Scholarship Grant for Foreign Students (Monbukagusho) for his $\mathrm{PhD}$ study. This work was partly supported by the Ministry of Agriculture, Forestry, and Fisheries of Japan (Genomics for Agricultural Innovation PMI-0010) and the Program for Promotion of Basic Research Activities for Innovative Biosciences (PROBRAIN), Japan and Grant-in-aid for Scientific Research from the Ministry of Education, Culture, Sports, Science and Technology, Japan to HK and RT (Grant-in-Aid for Scientific Research on Innovative Areas 23113009).

\section{REFERENCES}

Abe, A., Kosugi, S., Yoshida, K., Natsume, S., Takagi, H., Kanzaki, H., Matsumura, H., Yoshida, K., Mitsuoka, C., Tamiru, M., et al. (2012) Genome sequencing reveals agronomically important loci in rice using MutMap. Nature Biotechnol. 30, 174-178.

Apel, K., and Hirt, H. (2004) Reactive oxygen species: metabolism, oxidative stress, and signal transduction. Annu. Rev.
Plant Biol. 55, 373-399.

Arase, S., Fujita, K., Uehara, T., Honda, Y., and Isota, J. (2000) Light-enhanced resistance to Magnaporthe grisea infection in the rice Sekiguchi lesion mutants. J. Phytopath. 148, 197-203.

Bang, W., Kim, S., Ueda, A., Vikram, M., Yun, D., Bressan, R. A., Hasegawa, P. M., Bahk, J., and Koiwa, H. (2006) Arabidopsis carboxyl-terminal domain phosphatase-like isoforms share common catalytic and interaction domains but have distinct in planta functions. Plant Physiol. 142, 586-594.

Brodersen, P., Petersen, M., Pike, H. M., Olszak, B., Skov, S., Odum, N., Jorgensen, L. B., Brown, R. E., and Mundy, J. (2002) Knockout of Arabidopsis ACCELERATED. CELLDEATH11 encoding a sphingosine transfer protein causes activation of programmed cell death and defense. Genes Dev. 16, 490-502.

Dahmus, M. E. (1996) Reversible phosphorylation of the Cterminal domain of RNA polymerase II. J. Biol. Chem. 271, 19009-19012.

Dangl, J. L., Dietrich, R. A., and Richberg, M. H. (1996) Death don't have no mercy: cell death programs in plant-microbe interactions. Plant Cell 8, 1793-1807.

Dereeper, A., Guignon, V., Blanc, G., Audic, S., Buffet, S., Chevenet, F., et al. (2008) Phylogeny.fr: robust phylogenetic analysis for the non-specialist. Nucleic Acids Res. 36, W465-W469.

Hirose, M. E., and Manley, J. L. (2000) RNA polymerase II and the integration of nuclear events. Genes Dev. 14, 14151429

Ishikawa, A., Okamoto, H., Iwasaki, Y., and Asahi, T. (2001) A deficiency of coproporphyrinogen III oxidase causes lesion formation in Arabidopsis. Plant J. 27, 89-99.

Jabs, T., Dietrich, R. A., and Dangl, J. L. (1996) Initiation of runway cell death in an Arabidopsis mutant by extracellular superoxide. Science 273, 1853-1856.

Ji, H., Kim, S., Kim, Y., Kim, H., Eun, M., Jin, I., Cha, Y., Yun, D., Ahn, B., Lee, M. C., et al. (2010) Inactivation of the CTD phosphatase-like gene OsCPL1 enhances the development of the abscission layer and seed shattering in rice. Plant J. 61, 96-106.

Johal, G. S., Hulbert, S., and Briggs, S. P. (1995) Disease lesion mimic mutations of maize: A model for cell death in plants. Bioessays 17, 685-692.

Jung, Y., Lee, J., Agrawal, G., Rakwal, R., Kim, J., Shim, J., Lee, S., Jeon, J., Koh, H., Lee, Y., et al. (2005) The rice (Oryza sativa) Blast Lesson Mimic mutant, blm, may confer resistance to blast pathogens by triggering multiple defense associated signaling pathways. Plant Physiol. Biochem. 43, 397-406.

Jurkowski, G. I., Smith, R. K. Jr., Yu, I. C., Ham, J. H., Sharma, S. B., Klessig, D. F., Fengler, K. A., and Bent, F. A. (2004) Arabidopsis DND2, a second cyclic nucleotide-gated ion channel gene for which mutation causes the "defense, no death" phenotype. Mol. Plant-Microbe Interact. 17, 511520.

Kanzaki, H., Nirasawa, S., Saitoh, H., Ito, M., Nishihara, M., Terauchi, R., and Nakamura, I. (2002) Overexpression of the wasabi defensin gene confers enhanced resistance to blast fungus (Magnaporthe grisea) in transgenic rice. Theor. Appl. Genet. 105, 809-814.

Koiwa, H., Barb, A., Xiong, L., Li, F., McCully, G., Lee, B., Sokolchick, I., Zhu, J., and Gong, Z. (2002) C-terminal domain phosphatase-like family members (AtCPLs) differentially regulate Arabidopsis thaliana abiotic stress signaling, growth, and development. Proc. Natl. Acad. Sci. USA, 
99, 10893-10898.

Koiwa, H., Hausmann, S., Bang, W. Y., Ueda, A., Kondo, N., Hiraguri, A., et al. (2004) Arabidopsis C-terminal domain phosphatase-like 1 and 2 are essential Ser-5-specific Cterminal domain phosphatases. Proc. Natl. Acad. Sci. USA 101, 14539-14544.

Li, H., Ruan, J., and Durbin, R. (2008) DNA sequencing reads and calling variants using mapping quality scores. Genome Res. 18, 1851-1858.

Liu, D., Cheng, Z., Liu, G., Liu, G., Wang, Y., Zhao, X., and Zhu, L. (2003) Characterization and mapping of a lesion mimic in rice (Oryza sativa L. ). Chinese Sci. Bull. 48, 892-896.

Lorrain, S., Vailleau, F., Balague, C., and Roby, D. (2003) Lesion mimic mutants: keys for deciphering cell death and defense pathways in plants? Trends Plant Sci. 8, 263-271.

Mizobuchi, R., Hirabayashi, H., Kaji, R., Nishizawa, K., Yoshimura, A., Satoh, H., Ogawa, T., and Okamoto, M. (2002) Isolation and characterization of rice lesion-mimic mutants with enhanced resistance to rice blast and bacterial blight. Plant Sci. 163, 345-353.

Moeder, W., and Yoshioka, K. (2008) Mini-review: Lesion mimic mutants. Plant Signal Behav. 10, 764-767.

Nam, H. G. (1997) The molecular genetic analysis of leaf senescence. Curr. Opin. Biotechnol. 8, 200-207.

Noutoshi, Y., Ito, T., Seki, M., Nakashita, H., Yoshida, S., Marco, Y., Shirasu, K., and Shinozaki, K. (2005) A single amino acid insertion in the WRKY domain of the Arabidopsis TIRNBS-LRR-WRKY-type disease resistance protein SLH1 (sensitive to low humidity 1) causes activation of defense responses and hypersensitive cell death. Plant J. 43, 873888.

Qiao, Y., Jiang, W., Lee, J., Park, B., Choi, M., Piao, R., Woo, M., Roh, J., Han, L., et al. (2010) SPL28 encodes a clathrinassociated adapor protein complex 1 , medium subunit $\mu 1$ (AP1M1) and is responsible for spotted leaf and early senescence in rice (Oryza sativa). New Phytol. 185, 258-274.

Rakshit, S., Kanzaki, H., Matsumura, H., Rakshit, A., Fujibe, T., Okuyama, Y., Yoshida, K., Tamiru, M. O., Shenton, M., et al. (2010) Use of tilling for reverse and forward genetics of rice. In: The Handbook of Plant Mutation Screening: Mining of Natural and Induced Alleles (eds.: K. Meksem, and G. Kahl), pp. 187-197. Wiley-VCH Verlag GmbH \& Co. KGaA, Weinheim.

Sekiguchi, Y., and Furuta, T. (1965) On a rice mutant showing particular reaction to some spotting disease. Preliminary report. Ann. Phytopathol. Soc. Jpn 30, 71-72.

Suzuki, N., and Mitler, R. (2006) Reactive oxygen species and temperature stresses: A delicate balance between signaling and destruction. Physiol. Plant. 126, 45-51.

Takahashi, A., Kawasaki, T., Henmi, K., Shii, K., Kodama, O., Satoh, H., and Shimamoto, K. (1999) Lesion mimic mutants of rice with alterations in early signaling events of defense. Plant J. 17, 535-545.

Tian, B., Bevilacqua, C., Diegelman-Parente, A., and Mathews, M. (2004) The double-stranded-RNA-binding motif: Interference and much more. Nature Rev. Mol. Cell Biol. 5, 10131023

Ueda, A., Li, P., Feng, Y., Vikram, M., Kim, S., Kang, C. H., Kang, J. S., Bahl, J. D., et al. (2008) The Arabidopsis thaliana carboxyl-terminal domain phosphatase-like 2 regulates plant growth, stress and axing responses. Plant Mol. Biol. 67, 683-696.

Wu, C., Bordeos, A., Madamba, M. R. Z., Baraoidan, M., Ramos, M., Wang, G., Leach, J. A., and Leung, H. (2008) Rice lesion mimic mutants with enhanced resistance to diseases. Mol. Genet. Genomics 279, 605-619.

Xiong, L., Lee, H., Ishitani, M., Tanaka, Y., Stevenson, B., Koiwa, H., Bressan, R., Hasegawa, P., and Zhu, J. K. (2002) Repression of stress-responsive genes by FIERY2, a novel transcriptional regulator in Arabidopsis. Proc. Natl. Acad. Sci. USA 99, 10899-10904.

Yin, Z., Chen, J., Zeng, L., Goh, M., Leung, H., Khush, G. S., and Wang, G. (2000) Characterizing rice lesion mimic mutants and identifying a mutant with broad-spectrum resistance to rice blast and bacterial blight. Mol. Plant-Microbe Interact. 13, 869-876. 